\title{
Enabling Deep-Tissue Networking for Miniature Medical Devices
}

\author{
Yunfei Ma ${ }^{1}$, Zhihong Luo ${ }^{1}$, Christoph Steiger ${ }^{2,3,4}$, Giovanni Traverso ${ }^{2,3,4}$, Fadel Adib ${ }^{1}$ \\ ${ }^{1}$ MIT Media Lab $\quad{ }^{2}$ MIT Koch Institute ${ }^{3}$ Harvard Medical School $\quad{ }^{4}$ Brigham and Women's Hospital
}

\begin{abstract}
We present IVN (In-Vivo Networking), a system that enables powering up and communicating with miniature sensors implanted or injected in deep tissues. IVN overcomes fundamental challenges which have prevented past systems from powering up miniature sensors beyond superficial depths. These challenges include the significant signal attenuation caused by bodily tissues and the miniature antennas of the implantable sensors.

IVN's key contribution is a novel beamforming algorithm that can focus its energy toward an implantable device, despite its inability to estimate its channel or its location. We implement a multi-antenna prototype of IVN, and perform extensive evaluations via in-vitro, ex-vivo, and in-vivo tests in a pig. Our results demonstrate that it can power up and communicate with millimeter-sized sensors at over $10 \mathrm{~cm}$ depths in fluids, as well as battery-free tags placed in a central organ of a swine.

The implications of our new beamforming technology extend beyond miniature implantables. In particular, our results demonstrate that IVN can power up off-the-shelf passive RFIDs at distances of $38 \mathrm{~m}$, i.e., $7.6 \times$ larger than the operation range of the same RFIDs.
\end{abstract}

\section{CCS CONCEPTS}

- Networks $\rightarrow$ Cyber-physical networks; Mobile networks; Sensor networks;

\section{KEYWORDS}

Medical Implants, Deep-tissues, Power Delivery, RFID, Battery-free, Wireless Sensors

Permission to make digital or hard copies of all or part of this work for personal or classroom use is granted without fee provided that copies are not made or distributed for profit or commercial advantage and that copies bear this notice and the full citation on the first page. Copyrights for components of this work owned by others than the author(s) must be honored. Abstracting with credit is permitted. To copy otherwise, or republish, to post on servers or to redistribute to lists, requires prior specific permission and/or a fee. Request permissions from permissions@acm.org.

SIGCOMM '18, August 20-25, 2018, Budapest, Hungary

(C) 2018 Copyright held by the owner/author(s). Publication rights licensed to the Association for Computing Machinery.

ACM ISBN 978-1-4503-5567-4/18/08 . . \$15.00

https://doi.org/10.1145/3230543.3230566
ACM Reference Format:

Yunfei Ma, Zhihong Luo, Christoph Steiger, Giovanni Traverso, Fadel Adib. 2018. Enabling Deep-Tissue Networking for Miniature Medical Devices . In SIGCOMM '18: ACM SIGCOMM 2018 Conference, August 20-25, 2018, Budapest, Hungary. ACM, New York, NY, USA, 15 pages. https://doi.org/10.1145/3230543.3230566

\section{INTRODUCTION}

There is significant interest in bringing wireless networking capabilities to an emerging breed of in-vivo sensors and actuators [19, 37, 42]. In contrast to traditional implantable medical devices such as cardiac pacemakers, which are relatively large and have their own batteries, these new devices need to be much smaller: They are swallowed or injected into the human body and used for decoding brain circuits [21], delivering drugs [42], or monitoring internal human vital signs [61]. Despite the attention that these devices have received from the biomedical community, their networking capabilities are still very limited. This owes primarily to the very low power budget of these devices as well as their miniature form factor and biocompatibility requirements, which preclude incorporating batteries [27, 54].

Yet, empowering these sensors with networking capabilities would create a significant leap for in-vivo devices. Let us take the example of optogenetics, the recently developed biological technique that enables controlling neurons using light [21]. The vast majority of today's optogenetic manipulators that are implanted inside a brain need to be connected to an external source via a wire that passes through a drilled hole in a skull $[10,66]$. The wire is needed for communication and for powering up the manipulators. State-of-the-art proposals that do not require tethering insert a coil in the mammal's (e.g., mouse) skull and can only operate when the mammal is placed inside a charged chamber of $10 \mathrm{~cm}$ diameter that can deliver power to the implantable coil [50].

This paper presents IVN, ${ }^{1}$ a system that enables powering up and communicating with in-vivo battery-free biosensors and bioactuators. IVN introduces a multi-antenna technique to remotely power up millimeter-sized in-vivo sensors and communicate with them. To do so, it leverages frequencies in the sub-GHz radio frequency (RF) range (around $900 \mathrm{MHz}$ )

${ }^{1}$ IVN stands for In-Vivo Networking 
which has been demonstrated to achieve maximal power transfer through human tissues to in-vivo sensors [28, 50]. However, in contrast to existing solutions which can only deliver power at superficial depths or in highly constrained settings (e.g., mouse inside a cage), IVN can power up deep-tissue sensors and in realistic indoor environments.

The key challenge in delivering this vision arises from the ability to deliver sufficient power to the deep-tissue in-vivo sensors. This challenge arises from three main reasons:

- First, RF signals experience exponential attenuation as they propagate through the human body [23], which significantly limits the amount of power that can be delivered to an in-vivo sensor. Unfortunately, significantly boosting the transmitted power neither scales well nor is safe for human exposure [40, 57].

- Second, these miniature medical devices have very small form factor and as a result, they rely on millimeter-sized antennas, which have extremely low power harvesting efficiency [16, 49].

- Finally, because IVN relies on battery-free sensors, it cannot obtain channel feedback from these sensors (it needs to power them up in the first place), and hence cannot focus its energy in their direction using traditional means such as MIMO beamforming. This problem is further complicated by the fact that different human tissues have different RF propagation characteristics (due to changing dielectric constants), leading to complicated in-vivo channels that cannot be easily modeled.

To overcome these challenges, IVN introduces coherentlyincoherent beamforming (CIB), a new beamforming technology that can focus its energy to in-vivo sensors without prior channel knowledge. At a high level, instead of phaseencoding the transmitted signals from multiple antennas as in traditional beamforming, CIB frequency-encodes its signals. Such frequency encoding enables it to focus its energy on any point in 3D space, even in inhomogeneous media (e.g., multiple layers of tissues) and dense multipath due to reflections off different organs.

IVN incorporates CIB into a full system that can optimally deliver power to and communicate with deep-tissue biosensors and bioactuators. In designing CIB, we show how we can select frequency combinations that maximize power transfer to any point in 3D space. Our optimization function incorporates communication constraints of battery-free sensors including modulation depth, power stability, and adaptive duty cycling (as described in $\S 3$ ). We further extend IVN to deal with self-jamming caused by CIB transmissions through an out-of-band communication design (described in \$4).

We built a prototype of IVN and tested it with battery-free biosensors through in-vitro, ex-vivo, and in-vivo trials. Our prototype is built using USRP software radios [7] and tested using off-the-shelf battery-free millimeter-sized sensors [8]. Our experiments were approved by the Committee of Animal Care at MIT. Our results demonstrate the following:

- IVN can scale power delivery with the number of antennas without channel knowledge and through multiple layers of tissues. Moreover, in contrast to traditional beamforming/beamsteering techniques, IVN maintains its gains even in unknown media, and delivers up to $8.5 \times$ increase in power delivery over an optimized multi-antenna baseline.

- When tested with millimeter-sized battery-free sensors placed in liquid, IVN can power them up and communicate with them at depths of $11 \mathrm{~cm}$. In the absence of IVN, the sensors cannot be powered up beyond a superficial depth of few millimeters.

- We demonstrate that IVN can effectively power up and communicate with a deep-tissue battery-free sensor placed in a living pig's stomach.

- Finally, we show that IVN's beamforming approach is general and can have significant implications to the operation range of battery-free nodes like RFIDs. In particular, we demonstrate how the technique can achieve $7.6 \times$ increase in the range of powering up off-the-shelf RFIDs and demonstrate a reading range up to $38 \mathrm{~m}$ with RFIDs which would otherwise be able to only operate within $5 \mathrm{~m}$.

Contributions. We present the first system that can power and communicate with deep-tissue battery-free implants from meter-scale distances outside the body. Our key contribution is a new beamforming technology that can focus its energy toward an implantable device, despite blind channel conditions and significant attenuation from bodily tissues. Our design is implemented and evaluated to demonstrate communication with millimeter-sized sensors in living mammals.

\section{THE PROBLEM}

In this section, we explain the challenge in delivering energy to power up and communicate with miniature medical devices through deep tissues. We first describe the threshold problem in RF power harvesting, then highlight why overcoming this threshold is challenging for deep-tissue micro-implants.

\subsection{Primer on RF Power Harvesting}

We start by explaining the basic principle underlying RF power harvesting. To harvest energy, a battery-free sensor needs to convert RF signals in the environment into a DC (Direct Current) voltage. The component which allows the sensor to achieve such an RF-to-DC conversion is called an energy harvester (or rectifier).

A simplified schematic of an energy harvester is shown in the top left corner of Fig. 1. In its simplest form, the harvester consists of two capacitors $\left(C_{1}\right.$ and $\left.C_{2}\right)$ and two diodes $\left(D_{1}\right.$ and $D_{2}$ ). The diodes allow current to flow only in one direction (denoted by the triangle). In particular, when the voltage 


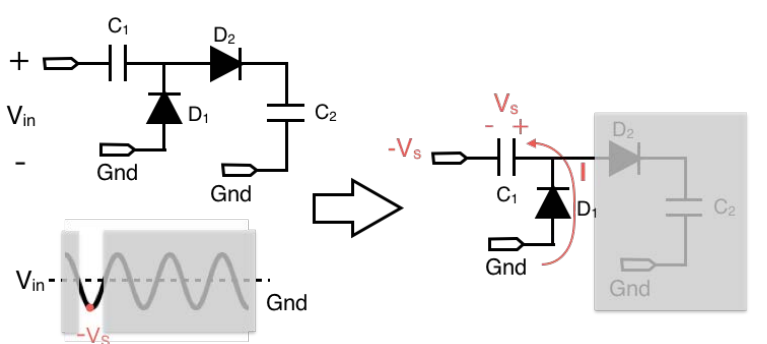

(a) Energy harvester operation in negative cycle.

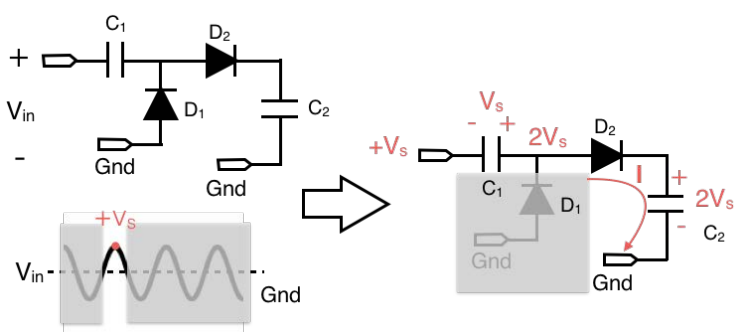

(b) Energy harvester operation in positive cycle

Figure 1-Energy harvesting circuit. The energy harvester utilizes a diode's rectifying effect to convert energy from RF to DC voltage.

across the diode is positive, it allows current to flow, but when the voltage across the diode is negative, it blocks the current.

To understand how the overall energy harvester works, we consider what happens when the RF signal alternates between its negative and positive half-cycles:

- Operation in negative half-cycle (Fig. 1(a)): In the negative half-cycle (i.e., $V_{i n}<0$ ), diode $D_{1}$ is $o n$ while diode $D_{2}$ is off. Hence, the current flows from the ground through $D_{1}$ and builds up charge in $C_{1}$. In the steady state, the voltage across $C_{1}$ is $V_{s}$, which denotes the amplitude of $V_{i n}$.

- Operation in positive half-cycle (Fig. 1(b)): In the positive half-cycle (i.e., $V_{i n}>0$ ), diode $D_{1}$ is off while diode $D_{2}$ is on. Hence, the current flows through $D_{2}$ and charges $C_{2}$. In the steady state, the voltage across $C_{2}$ is $2 V_{s}$, since it is the summation of the peak of $V_{i n}$ and the built up voltage across $C_{1}$ during the negative half-cycle.

The above description is simplified in two aspects:

- First, our schematic shows a single-stage power harvester. Today's power harvesters are constructed in multi-stage structure, with each stage multiplying the voltage of the previous one. Hence, the voltage at the output of an $N$-stage harvester is $N V_{s}$.

- Second, the description assumes an ideal power harvester where all the input voltage can be harvested. In practice, however, the performance of the energy harvester declines dramatically as the input voltage $V_{s}$ decreases. This is due to the diode threshold effect, which we explain next.

\subsubsection{Threshold Effect}

In our previous discussion, we assumed that a diode is on whenever the voltage across it is positive, and that it is off
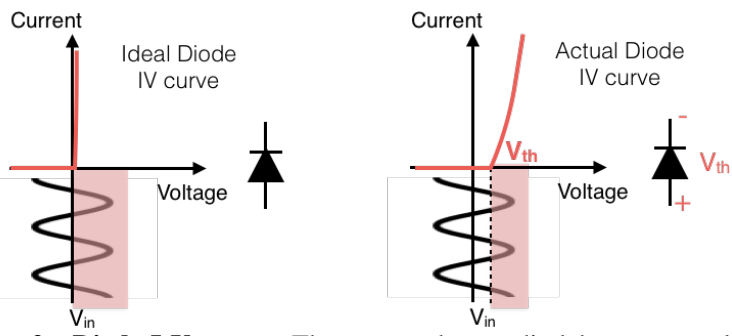

Figure 2-Diode I-V curves. The curves show a diode's current-voltage relationship in the ideal (left curve) and realistic (right curve) scenarios.
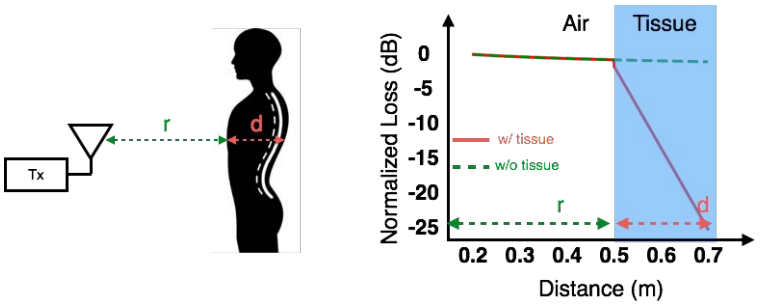

Figure 3-Signal power loss in tissues vs. in air. The right figure plots the normalized loss (in log scale) as a function of distance in air and tissues.

whenever the voltage across it is negative. The left plot of Fig. 2 shows this behavior by plotting the current (I) versus voltage $(\mathrm{V})$ curve across an ideal diode.

\subsection{RF Power Harvesting in Deep Tissues}

Overcoming the threshold voltage in deep tissue implantables is challenging for two reasons. The first is the attenuation of RF signals as they propagate in biotissues, and the second is their miniature form factor. In what follows, we describe both of these challenges in detail.

Practical diodes, however, do not exhibit this ideal behavior. In particular, in order to conduct a current through a diode, one must overcome an energy barrier, which is reflected in the threshold voltage. Said differently, the input voltage has to be larger than a threshold voltage $V_{t h}$ to turn on a diode. Whenever the voltage drops below the threshold, the diode switches off. As a result, the voltage that accumulates in the negative cycle is equal to $V_{s}-V_{t h}$, and the voltage that can be eventually achieved across $C_{2}$ is $2\left(V_{s}-V_{t h}\right)$. When extending to an $N$-stage energy harvester, the maximum output voltage $V_{D C}$ can be written as:

$$
V_{D C}=N\left(V_{s}-V_{t h}\right)
$$

As can be seen from Eq. 1, due to the voltage barrier $V_{t h}$, the energy harvester is significantly more efficient with a large input voltage than with a small input voltage. In fact if $V_{s}<V_{t h}$, the energy harvester cannot harvest any energy. For standard integrated circuits (IC) process, the threshold voltage is usually between $200 \mathrm{mV}$ and $400 \mathrm{mV}$ [22, 26, 41].

Indeed, it is this threshold voltage that places a fundamental limit on the operation of RF power harvesters. For example, a passive RFID today can only be powered up if it is within 


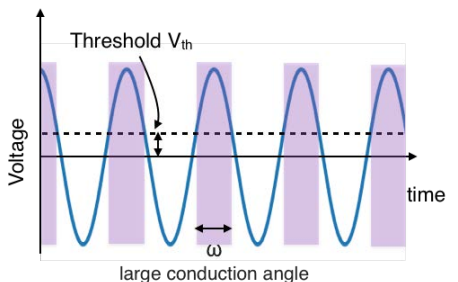

(a) Threshold impact at small distance in air

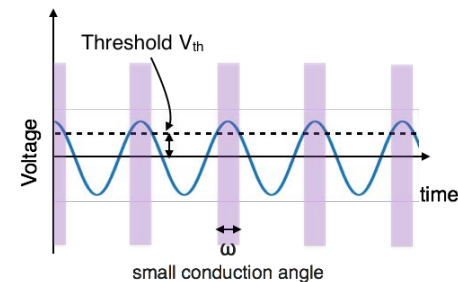

(b) Threshold impact at large distance in air

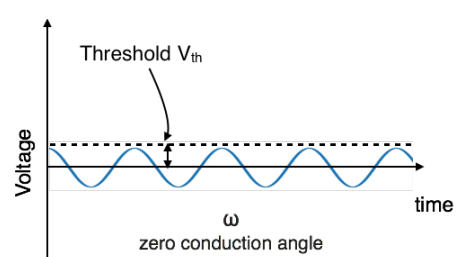

(c) Threshold impact in body

Figure 4-Impact of the threshold effect of RF power harvesting. The figure plots the voltage vs time when the sensor is (a) close to the transmitter in air, (b) at shallow depth in tissues, and (c) in deep tissues. The figure highlights the conduction angle (i.e., when the diode is on) in purple.

5-10 $\mathrm{m}$ from the RFID reader [51]. Beyond this range, the received power cannot generate enough energy to overcome the diode threshold voltage.

\subsubsection{RF Propagation in Tissues}

RF signals traveling from air into biotissues experience two sources of attenuation, which are depicted in Fig. 3:

- The first source of attenuation arises from the reflection of RF signals at the air-tissue boundary. Due to this reflection, only a fraction of the incident signal traverses the boundary. For RF signals in the $1 \mathrm{GHz}$ range, this results in a loss of around 3-5 dB [36].

- The second and more significant source of attenuation arises from the exponential loss due to propagation through the tissue. For example, RF signals in the low-GHz range experience a loss ranging from 2.3 to $6.9 \mathrm{~dB} / \mathrm{cm}$ considering tissue a dielectric constant of 50 and a conductivity of 1 to $3 \mathrm{~S} / \mathrm{m}[36,39]$. This translates to a loss of 11.5 to $35.4 \mathrm{~dB}$ at a depth of $5 \mathrm{~cm}$.

Mathematically, let us denote the distance between the body and transmit antenna by $r$ and the depth inside the tissue by $d$ (with $d \ll r$ ), as shown in Fig. 3. We can express the overall amplitude of the electric field $E$ in the biotissue as:

$$
|E|=\frac{T A}{r} e^{-\alpha d}
$$

where $A$ is the amplitude of the transmitted signal, $T$ is the transmittance coefficient (i.e., the fraction that traverses the air-tissue boundary), and $\alpha$ is the attenuation constant of the RF signal in the tissue. For different tissues, $\alpha$ can vary between $13 \mathrm{~m}^{-1}$ and $80 \mathrm{~m}^{-1}$ [39].

Note from Eq. 2 that if the signal travels entirely in air, then the signal attenuation is only inversely proportional to the travel distance. However, once the signal starts propagating in tissues, the exponential term dominates. Also note that the above equation describes the electric field, while the received power is proportional the $E^{2}$, and hence degrades quadratically in $r$ and exponentially in $d$.

\subsubsection{Miniature Antenna Size}

The second challenge for harvesting energy in deep tissues arises from the miniature form factor of microimplants. In particular, the desire to implant or inject medical devices into the brain, blood stream, or in deep tissues places stringent constraints on their overall size [27, 54]. This, in turn, constrains the size of their antennas, often to millimeter-scale (or sub-millimeter) dimensions [28].

Yet, the ability of an antenna to harvest RF power is directly related to its size. In particular, the receive power $P_{L}$ that may be harvested by the energy-harvesting circuit can be expressed as a function of the electric field $E$ and the effective antenna area $A_{\text {eff }}$ as:

$$
P_{L}=\frac{E^{2}}{\eta} A_{e f f}
$$

where $\eta$ denotes the wave impedance, which is a function of the medium.

As per Eq. 3, the amount of energy harvested is directly proportional to the effective area of the antenna. As an analogy, a passive RFID's range uniformly increases with its size (assuming proper matching) [51]. This places a stringent constraint on the power-harvesting efficiency of sensors with antenna dimensions smaller than $1 \mathrm{~cm}$.

\subsection{Summary}

In summary, the challenge of in-vivo battery-free networking arises from two competing factors. On one hand, due to the threshold effect, the energy harvesting efficiency is highly sensitive to the signal amplitude. On the other hand, the exponential tissue loss and miniature antenna form factor cause significant attenuation.

To understand how these two factors play out, Fig. 4 illustrates what happens when a sensor is moved from air into deep-tissues. We focus on the sensor's conduction angle $\omega$, which represents the time interval when the diode is on. When the sensor is in air and close to the RF power source (Fig. 4(a)), the signal is well above the threshold voltage, and we have a large conduction angle. In this case, most of the input RF energy can be effectively converted to DC voltage, and the overall energy harvesting efficiency is high. When the sensor is placed at shallow depths in a tissue (Fig. 4(b)), the input signal amplitude is attenuated but may remain above the threshold voltage. The conduction angle is smaller than in Fig. 4(a). In this case, the percentage of the input signal power that can be effectively used is small, which decreases the overall energy harvesting efficiency, but the system may still operate (e.g., by duty cycling the sensor's operation so 


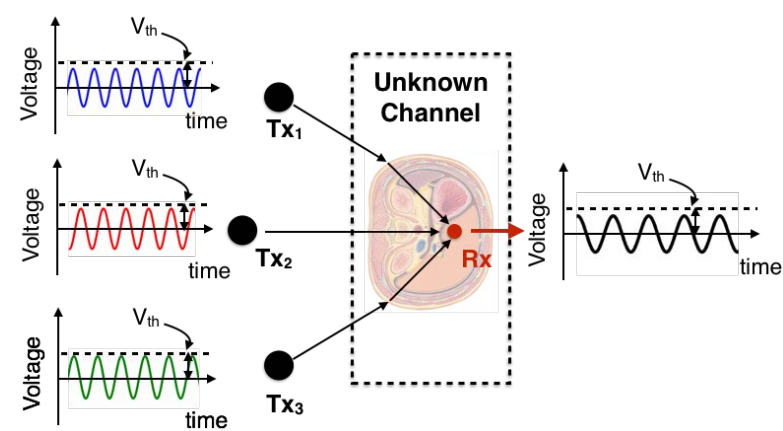

(a) Traditional beamforming under unknown channel conditions.

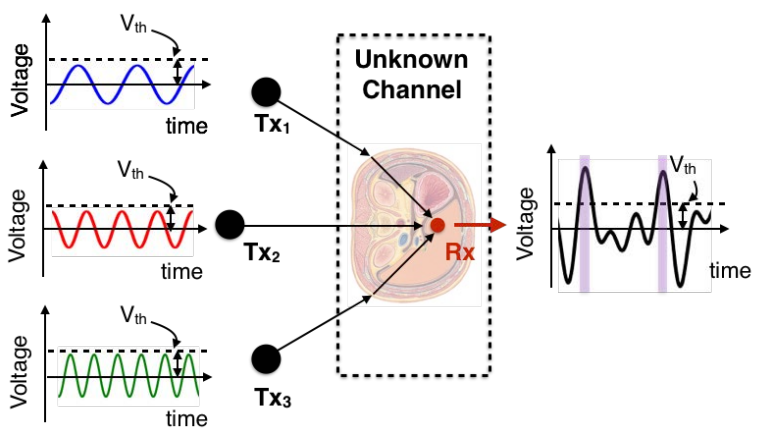

(b) IVN's CIB technique under unknown channel conditions.

Figure 5-Comparing traditional beamforming to IVN's CIB under blind channel conditions. (a) Traditional beamforming employs coherent frequencies which are unlikely to combine constructively without channel feedback. (b) IVN's CIB transmits synchronized commands at multiple incoherent carriers; this results in boosting the peak power and overcoming the threshold effect at the sensors' energy harvester without the need for channel information.

that it may accumulate sufficient energy before communication or actuation). However, when the sensor is placed deep inside a tissue (Fig. 4(c)), even the peak value of the input voltage drops below the threshold. In this scenario, the conduction angle becomes zero and no energy can be harvested.

\section{OUR DESIGN}

IVN is a system that enables wireless power delivery and communication with deep-tissue miniature medical devices. Its key innovation is a new beamforming algorithm that can focus its energy toward an in-vivo sensor. The focused energy allows the sensor to overcome the threshold voltage despite RF attenuation and despite the sensor's miniature size.

In this section, we highlight the need for this new beamforming algorithm for in-vivo battery-free sensors, and describe IVN's algorithm.

\subsection{Beamforming for In-Vivo Battery-Free Sensors}

Similar to standard beamforming algorithms, IVN leverages multiple antennas to focus its energy toward an in-vivo sensor. Fig. 5(a) shows the architecture of a standard beamformer with multiple transmit antennas. The signals transmitted from the different antennas traverse slightly different paths before arriving at the sensor. Hence, they experience different channels. The goal of a beamformer is to precode these transmitted signals so that they constructively interfere at the receiver, maximizing the received energy.

Mathematically, if we denote signal transmitted from antenna $i$ by $x_{i}$, and the channel experienced by that signal as $h_{i}$, we can express the signal received at the in-vivo sensor as:

$$
y=\sum_{i} h_{i} x_{i}
$$

Hence, in order to maximize the received energy, IVN should estimate the channels $h_{i}$ and set $x_{i}=h_{i}^{*}$.

Unfortunately, channel estimation is infeasible in the context of in-vivo battery-free networking for two reasons:
- Tissue inhomogeneity: As Fig. 5 shows, RF signals traveling from air to a sensor traverse different media, including multiple layers of tissues (including skin, fat, muscles, etc.). These signals may also experience multipath as they reflect off different organs before arriving at the sensor. This makes it intractable to predict $h_{i}$. In contrast, in a homogeneous medium, one can easily model the propagation characteristics and predict the relationship between different $h_{i}$ 's.

- Battery-Free Nature: In principle, one could overcome this challenge by directly estimating the channel between the transmitter and the receiver. However, channel estimation requires powering up the battery-free sensor in the first place, which is infeasible when the sensor is in deep-tissue.

Hence, IVN must beamform under blind channel conditions.

\subsection{Coherently-Incoherent Beamforming}

To overcome the above challenges, IVN introduces a novel beamforming technology: coherently-incoherent beamforming (CIB). Similar to traditional beamforming, CIB employs multiple antennas. However, CIB is unique in that it can focus energy from its antennas even under blind channel conditions. As indicated in its name, CIB has two key properties:

- First, CIB's communication process is coherent, meaning that the commands transmitted from all the antennas are the same, and all antennas transmit their commands at the exact same time (synchronously). This is important because battery-free sensors decode by detecting energy levels; hence, the transmissions need to be synchronized in order for the sensors to observe the same energy envelopes across the different transmit antennas.

- Second, CIB's wireless channel is designed to be incoherent, meaning that the frequencies transmitted from the antennas are different. The frequency discrepancies emulate a time-varying channel, and IVN exploits the the time-varying characteristics to enable power delivery even under blind channel conditions as we explain next. 


\subsection{CIB's Basic Formulation}

Next, we formally define the operation of a CIB beamformer. Consider a beamformer with $N$ transmit antennas. For simplicity, let us assume that each antenna sends a single frequency $f_{i}$ (In practice, IVN can modulate its command atop the carrier frequency). Because the frequencies are generated by different oscillators (PLLs), the transmitted signals have a random initial phase offset $\theta_{i}$. At the receiver, each signal will experience a phase rotation $\phi_{i}$ which depends on the channel from the transmit antenna. Hence, we can write the received signal at the receiver as:

$$
y(t)=\sum_{i=1}^{N} e^{j\left(2 \pi f_{i} t+\theta_{i}+\phi_{i}\right)}=\sum_{i=1}^{N} e^{j\left(2 \pi f_{i} t+\beta_{i}\right)}
$$

Since $\theta_{i}$ is sampled uniformly at random, $\beta_{i}=\theta_{i}+\phi_{i}$ is sampled from a uniform distribution over $[0,2 \pi]$.

\subsection{Overcoming the Threshold Limit}

To understand why CIB's formulation allows us to overcome the threshold limit, let us compare its operation to that of a traditional beamformer under blind channel conditions. As shown in Fig. 5(a), in traditional beamforming, the signals transmitted from different antennas (shown in blue, red, and green) all have the same frequency. Hence, the combined signal that arrives at the sensor will also have the same frequency, and the amplitude of that frequency will depend on the (unknown) channels. In such a scenario, the beamformer will always encounter blind spots, i.e., locations inside the body where the signals will add up destructively and hence be unable to deliver power.

Now let us consider what happens with CIB. As shown in Fig. 5(b), the signals transmitted from its different antennas (shown in blue, red, and green) all have different frequencies. Hence, the signal that arrives at the sensor (shown in black) will have a time-varying envelope. This time-varying envelope is desirable for multiple reasons:

- First, it provides an opportunity to overcome the threshold voltage (as shown in Fig. 5(b)). In particular, since the different signals have different frequencies, their phases will shift with respect to each other over time, providing opportunities for constructive (and destructive) interference at any given location. This allows the sensor to harvest energy during periods where the voltage is larger than the threshold (marked by pink regions).

- Second, if we consider another set of $\beta_{i}^{\prime} s$ (i.e., another point inside the body or another combination of initial phase offsets), the sensor will also have opportunities for constructive interference, which may peak above the threshold voltage. This means that CIB can charge sensors at different points inside the human body using the same set of frequency combinations, albeit each sensor experiences the peak voltage at a different point in time.

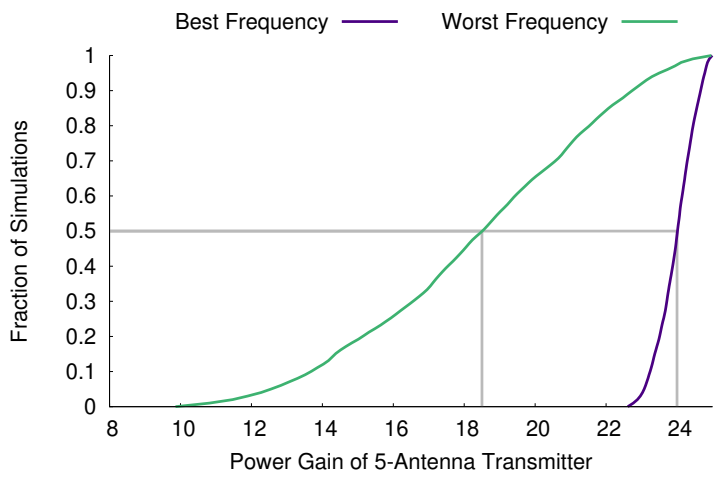

Figure 6-CIB's power gain from a 5-antenna transmitter. The figure plots the CDFs of the best (purple) and worst (green) frequency combinations from monte-carlo simulations across different channel conditions.

Note that the average received energy is the same across both encoding schemes (phase-based and frequency-based). The key advantage of frequency-based encoding in CIB, however, is that it can achieve higher peaks, which enables the in-vivo sensor to overcome its threshold voltage. Effectively, this beamforming technique focuses its energy over a short period of time and duty cycles the energy.

In addition, we make the following observations about CIB:

- The maximum achievable peak in CIB is $N$ (when the signals from all the antennas constructively interfere at some point in time). Hence, the maximum power gain is $N^{2}$. Note that even if we keep the same power budget (i.e., multiply the amplitude of the transmitted signal from each antenna by $1 / \sqrt{N}$ ), CIB would still provide a $N \times$ power gain. This gain matches that of traditional beamforming, but CIB achieves it even under blind channel conditions.

- One might wonder if CIB could be employed as a replacement to prior beamforming approaches (e.g., antenna-arraybased beamforming). The answer depends on the problem domain. In particular, CIB addresses different constraints and harnesses different opportunities in comparison to other beamforming technologies. For example, MIMO or antenna array beamforming is desirable in wireless communication, as it enables sustaining high signal-to-noise ratio (SNR) and hence throughput during an entire communication session, but it requires channel estimates. In contrast, CIB can deliver the peak power to any point in 3D space, but over a short period of time and in blind channel conditions. Hence, it is more appropriate for powering up battery-free sensors when the channel is unknown.

\subsection{Impact of Frequency Selection}

One may ask whether an arbitrary frequency selection for CIB would enable it to achieve the $N^{2}$ peak power gain. To understand the impact of CIB's frequency selection, we run monte-carlo simulations, where we study the behavior of two random sets consisting of 5 frequencies under random 
channel conditions. For each sampling point, we choose a random initial phase $\beta_{i}$ for each frequency in order to emulate the blind channel conditions. We then measure the peak power from the maximum value of $y(t)$ over time.

Fig. 6 plots the CDFs of the peak power gains from the two different frequency sets. The figure shows that the two sets exhibit drastically different performance. In particular, the blue curve corresponds to a set which can achieve $90 \%$ of the optimal performance across all possible channel conditions. On the other hand, the green curve shows a frequency selection where across more than half the channel conditions, the power gain is smaller than $75 \%$ of the optimal performance. Hence, the performance of CIB is highly dependent on the selected frequency combination.

\subsection{Objective Function}

In order to determine the optimal frequency combination that yields the highest peak power, we formulate the task as an optimization problem:

$$
\underset{f_{1}, \ldots, f_{N}}{\arg \max _{\boldsymbol{\beta}}}\left[\max _{t}\left|\sum_{i=1}^{N} e^{j\left(2 \pi f_{i} t+\beta_{i}\right)}\right|\right]
$$

where $\boldsymbol{\beta}=\left(\beta_{1}, \beta_{2}, \ldots, \beta_{N}\right)$ and $\mathbf{E}[\cdot]$ indicates expectation. Said simply, our goal is to find the frequency selection that maximizes the expected peak power over all possible channel conditions. We note that this optimization problem is not convex and hence it is not easily solvable.

Observe that the optimization problem in Eq. 6 does not depend on the absolute frequency values $f_{i}$ but rather on the frequency offsets. This is because $\left|\sum_{i=1}^{N} e^{\left(j\left(2 \pi f_{i} t+\beta_{i}\right)\right)}\right|=$ $\left|\sum_{i=1}^{N} e^{\left(j\left(2 \pi \Delta f_{i} t+\Delta \beta_{i}\right)\right)}\right|$ where $\Delta f_{i}=f_{i}-f_{1}$ and $\Delta \beta_{i}=\beta_{i}-\beta_{1}$. Hence, solving the optimization problem is equivalent to finding the optimal set of frequency differences $\Delta f_{i}$.

In addition to maximizing the peak power, CIB's frequency selection has to satisfy the following two constraints that arise from the communication nature of the problem:

(a) Cyclic operation: In health sensing applications, it is desirable to continuously monitor physiological conditions. Assume that we would like to obtain a sensor response every $T$ seconds. Then, to ensure that CIB delivers its peak power every $T$ seconds, we must satisfy two constraints:

- $T$ must be divisible by all $1 / \Delta f_{i}$ for all $i=2 \ldots N$.

- The maximization function is computed over $0<t<T$

Without loss of generality, we can assume that $T=1 \mathrm{~s}$, and hence restrict ourselves to integer $\Delta f$ 's.

(b) Query amplitude flatness: The second constraint arises from the operation of a backscatter sensor. Specifically, backscatter sensors decode the downlink query command by envelope detection. As a result, they can tolerate only a small fluctuation in the amplitude of their received signal. CIB, however, introduces amplitude variation by design. Hence, in order for the backscatter nodes to correctly decode, we must ensure that the received waveform satisfies the fluctuation constraint. Formally, if we define $A_{\max }$ as the largest amplitude and $A_{\min }$ as the minimum amplitude, we need to satisfy the following condition:

$$
\left(A_{\max }-A_{\min }\right) / A_{\max } \leq \alpha
$$

where $\alpha$ is defined as the percentage fluctuation. One should ensure that $\alpha<0.5$. This is because the sensor's energy detector uses half the amplitude difference as the decoding threshold to differentiate between bits 0 and 1 .

Now let us consider the case of envelope degradation from the highest possible peak. Define $Y(t)=\left|\sum_{i=1}^{N} e^{\left(j\left(2 \pi \Delta f_{i} t+\Delta \beta_{i}\right)\right)}\right|$ as the envelope of beamforming signal and assume that at time $t_{0}$, the phases of all the carriers are perfectly aligned, giving the highest possible peak. That is $2 \pi \Delta f_{i} t_{0}+\Delta \beta_{i} \equiv 0$ $(\bmod 2 \pi)$ for $i=1 \ldots N$, so that $Y\left(t_{0}\right)=N$. Let the $\Delta t$ denote the duration of the query command. Then, at $t_{0}+\Delta t$, if $\Delta t$ is relatively small, we approximate $Y(t)$ to the first order Taylor series expansion as:

$$
Y\left(t_{0}+\Delta t\right) \leq \sum_{i=1}^{N} \cos \left(2 \pi \Delta f_{i} \Delta t\right) \leq N-2 \pi^{2} \Delta t^{2}\left(\sum_{i=1}^{N} \Delta f_{i}^{2}\right)
$$

from Eq. 7, we have $\frac{Y\left(t_{0}\right)-Y\left(t_{0}+\Delta t\right)}{Y\left(t_{0}\right)} \leq \alpha$, which leads to

$$
\frac{1}{N} \sum_{i=1}^{N} \Delta f_{i}^{2} \leq \frac{\alpha}{2 \pi^{2} \Delta t^{2}}
$$

Eq. 9 shows that the mean square of $\Delta f_{i}$ should be constrained to allow more smooth transition at the peak power. For a typical RFID reader's query, $\Delta t \approx 800 \mu \mathrm{s}$. Hence, the root mean square of $\Delta f_{i}$ should be less than $199 \mathrm{~Hz}$.

Putting the above constraints together, the problem in Eq. 6 can be solved by the following two steps:

- First, we select the optimal center frequency $f_{1}$ based on the system hardware constraints such as antenna impedance matching, power amplifier bandwidth, etc.

- Second, we then choose the optimal frequencies $f_{i}=$ $f_{1}+\Delta f_{i}$ by solving the following constrained heuristic optimization problem:

$$
\begin{aligned}
\max _{\Delta f_{2}, \ldots, \Delta f_{N} \in \mathrm{N}} \mathbf{E}_{\boldsymbol{\beta}} & {\left[\max _{0 \leq t \leq 1}\left|1+\sum_{i=2}^{N} e^{j\left(2 \pi \Delta f_{i} t+\beta_{i}\right)}\right|\right] } \\
\text { s.t. } & \frac{1}{N} \sum_{i=2}^{N} \Delta f_{i}^{2} \leq \frac{\alpha}{2 \pi^{2} \Delta t^{2}}
\end{aligned}
$$

To solve the above optimization function, IVN performs a one-time monte-carlo simulation (as we explain in $\$ 5$ ). ${ }^{2}$ Once optimal frequency selection has been determined, IVN modulates its downlink commands atop the carrier frequencies and sends them synchronously from multiple antennas. ${ }^{2}$ Note that this simulation needs to be solved only once, since it optimizes
for all channel conditions. 


\subsection{Further Discussion of CIB's Design}

Below, we describe some of the powerful features of CIB as well as potential extensions of its core design:

- Powering and communicating with multiple sensors: Our above discussion focused on a single sensor. However, in principle, IVN's communication can seamlessly scale to multiple in-vivo sensors. This is because a CIB beamformer scans 3D space through its time-varying channel. In order to avoid collision between multiple sensors, IVN can leverage a variety of techniques from standard backscatter communications. For example, it may incorporate a select command into its query [3], specifying the identifier of the sensor it wishes to communicate with. If this results in elongating the query command, it can incorporate this into the $\Delta t$ constraint of Eq. 10 .

- Robustness to multipath and mobility: CIB's design is inherently robust to phase changes caused by channel variations, including those caused by multipath, medium homogeneity, and mobility. However, the formulation of Eq. 10 assumes that all the frequencies lie within the coherence bandwidth (i.e., it does not account for frequency-selective fading). In some scenarios, all the frequencies may experience multipath fading. While CIB can still provide the same gain in these scenarios, the overall power delivered will be lower. An extension of this design may adaptively hop the center frequency to a different band to improve performance.

- Optimizing power transfer with depth knowledge: One might wonder why CIB chooses to optimize for the peak power rather than maximizing the conduction angle (depicted in Fig. 5), which could improve the overall power transfer efficiency. The challenge with maximizing conduction angle, however, is that it requires a priori knowledge of the attenuation. For example, if IVN underestimates the attenuation, then it might not be able to deliver sufficient power to excite the sensor. In practice, one may extend CIB into a two-stage design. The first stage involves a discovery process where it optimizes for peak power; then, once it has determined the overall attenuation, it can switch to a steady stage where it maximizes the conduction angle.

\section{JAMMING-FREE COMMUNICATION}

A key challenge in enabling communication in the presence of CIB beamforming arises from self-jamming. Specifically, since the transmitted signals may combine constructively both at the sensor and at IVN's receive antenna, they may saturate its receiver and prevent it from decoding the backscatter sensor's response.

To overcome this jamming problem, IVN employs an out-of-band reader, which enables it to filter out the selfjamming from CIB's transmit antennas. The out-of-band reader is inspired by recent work on two-frequency excitation [46]. Specifically, since backscatter modulation is

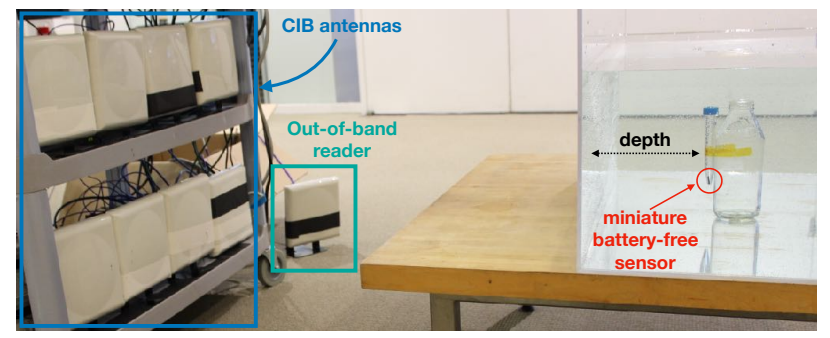

Figure 7-IVN's setup with a miniature battery-free sensor in liquid. A sample experimental setup where the millimeter-sized RFID was placed inside a tank filled with water.

frequency-agnostic, once a backscatter node (e.g., RFID) is powered up, a reader may sense and decode its response at a different carrier frequency. Note that in contrast to past work which uses this technique to enable wideband sensing for localization, our out-of-band reader leverages this technique to reduce self-interference. In particular, IVN's out-of-band reader transmits and receives coherently at a carrier frequency that is different from those employed by CIB's transmitters. This enables the reader to filter out the jamming signal and decode the response out-of-band.

\section{IMPLEMENTATION}

We describe the different components of our prototype implementation of IVN, shown in Fig. 7.

(a) CIB Beamformer: We built IVN's multi-antenna beamformer on USRP N210 software radios [7] with SBX daughterboards [5]. The output of each USRP is fed into a HMC453QS16 power amplifier, whose 1-dB compression point is $30 \mathrm{dBm}$. Each amplifier is then connected to MT242025, a 7 dBi RHCP RFID antenna [4]. Unless otherwise noted, most of our evaluation is performed with eight USRPs. The USRPs are all connected to a CDA-2900 Octoclock with a $10 \mathrm{MHz}$ reference clock and a PPS synchronization pulse signal [2]. At the backend, all the USRPs are connected via Ethernet cables to a 64-bit machine running Ubuntu 16.04.

We implemented the beamforming algorithm and concurrent data communication directly into the USRP's UHD driver in $\mathrm{C}++$. The communication follows the standard RFID protocol and is adapted from [34]. Each USRP is programmed to transmit at a different frequency. The center carrier is chosen at $915 \mathrm{MHz}$ and the relative frequency differences $\Delta f_{i}(i=1 \ldots 10)$ are $0,7,20,49,68,73,90,113,121$ and $137 \mathrm{~Hz}$, respectively. ${ }^{3}$ The USRPs synchronously transmit their commands at their respective frequencies. Frequency selection is performed as per our description in $\S 3.6$. Note that since USRPs cannot stably generate small frequency offsets,

\footnotetext{
${ }^{3}$ We obtain these frequencies using a one-time simulation. The overall simulation time in MATLAB on a standard workstation with an Intel Core i7 processor is less than 5 mins.
} 
In-Vivo Networking

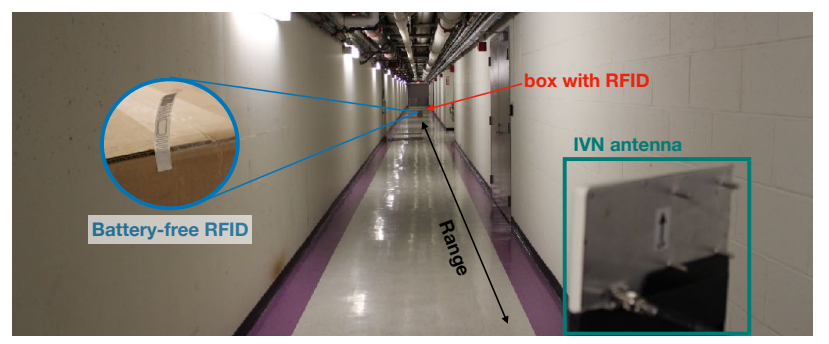

Figure 8-IVN's setup for long-range communication. Our setup for testing IVN's communication range with a battery-free RFID in line-of-sight.

we soft-coded these offsets directly into the complex numbers before sending them to the USRP.

(b) Out-of-band Reader. IVN employs an out-of-band reader to enable jamming-free communication. The reader is implemented on two USRP N210 software radios with SBX daughterboards, connected to MT-242025 antennas[4]. One USRP is used for transmitting and one for receiving. To avoid jamming from IVN's beamformer, the reader operates at a different center frequency than the beamformer, namely at $880 \mathrm{MHz}$. The received signal is filtered using a high-rejection SAW filter to mitigate jamming from the beamformer.

The reader is time-synchronized with the beamformer and synchronously transmits its same commands. This ensures that the commands do not collide at the sensor, and that the reader's signal does not compromise the modulation depth. The receiver measures the backscatter sensor's response as it replies to the beamformer's command. To compensate for the large attenuation in tissues, the reader averages responses over 1-second intervals. This constitutes the period of CIB's envelope, and allows the IVN to coherently combine the backscatter responses to boost the SNR. The reader then uses a standard RFID decoder in MATLAB adapted from [34] and operates on the averaged signal.

(c) Battery-free Sensors: We evaluate IVN with two types of commercial battery-free backscatter nodes. The first is a standard RFID tag, the Avery Dennison AD-238u8 [1]. The tag measures $1.4 \mathrm{~cm} \times 7 \mathrm{~cm}$ and is shown in Fig. 8. The second is a miniature RFID tag, the Xerafy Dash-On XS RFID tag [8]. The miniature tag measures $1.2 \mathrm{~cm} \times 0.3 \mathrm{~cm} \times 0.22 \mathrm{~cm}$ and is shown in Fig. 7. In all our liquid, in-vivo, or ex-vivo evaluations, the tag is placed in a test tube as shown in Fig. 7. This ensures that the medium immediately surrounding the tag is matched to its antenna impedance. Note that this is not a requirement if the antenna is designed to match for the surrounding medium [49].

\section{EVALUATION \& RESULTS 6.1 In-Vitro and Ex-Vivo Evaluation}

We start by reporting our results from in-vitro and ex-vivo evaluations. These experiments include tests performed in simulated fluids and in animal tissues.
SIGCOMM '18, August 20-25, 2018, Budapest, Hungary

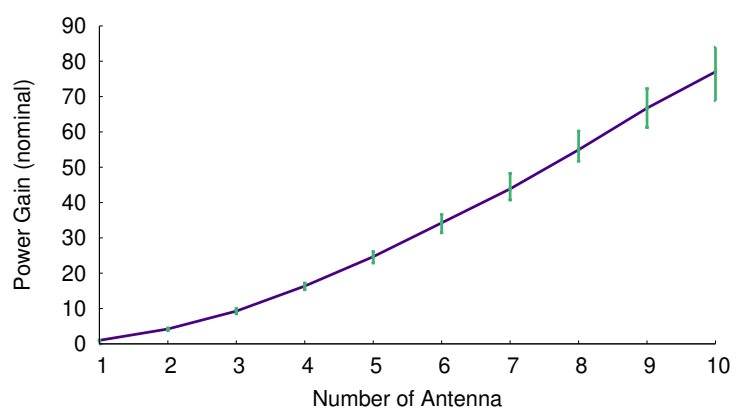

Figure 9-Gain versus number of antennas. Peak power gain is the ratio of the peak power from the beamformer to the peak power from a signal antenna to the same location. Error bars indicate the $10^{t h}$ and $90^{t h}$ percentiles.

\subsubsection{IVN's Power Gain in Blind Channel Conditions}

Recall that the goal of IVN's beamformer is to boost the peak power received at a sensor so that it overcomes its threshold voltage. So, to evaluate the effectiveness of IVN, we compare the peak power with and without its beamforming algorithm.

We perform our peak power measurements using a dedicated USRP receiver with an RFX900 antenna. ${ }^{4}$ The USRP collects measurements for 2-second intervals (i.e., well over the duty cycling period of the beamformer). We post-process the captured signal in MATLAB and identify its maximum peak. We then compute the power gain as the square of the ratio between the peak amplitudes with and without CIB.

(a) Gain vs Number of Antennas: We perform our first set of experiments in a setup similar to Fig. 7. In these experiments, we fixed the distance between the beamformer and the edge of the container to around $0.5 \mathrm{~m}$. We conduct $150 \mathrm{ex}-$ perimental trials in total. In each trial, we change the location and orientation of the receive antenna and compute the power gain as described above.

Fig. 9 plots the median, $10^{\text {th }}$, and $90^{\text {th }}$ percentile of nominal power gain as a function of the number of beamformer antennas. We make the following observations:

- The power gain monotonically increases as the number of antennas increases from 1 to 10 , and achieves a gain as high as $85 \times$ with 10 antennas. This demonstrates that IVN's beamformer can indeed boost the peak power of the received signal even in blind channel conditions.

- IVN cannot always achieve the optimal peak power gain of $N^{2}$. This is because the frequency combination is not guaranteed to always achieve the highest maximum as shown in Fig. 6.

(b) Gain vs Distance and Orientation: Next, we would like to understand the impact of distance and orientation of the receive antenna on the peak power gain. Hence, we fix the number of transmit antennas to 10 and repeat the above experiments at different locations and orientations. Figs. 10(a)

\footnotetext{
${ }^{4}$ The antenna is connected to the USRP with a shielded wire.
} 


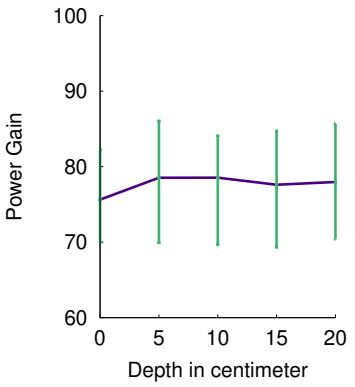

(a) Power Gain vs Depth.

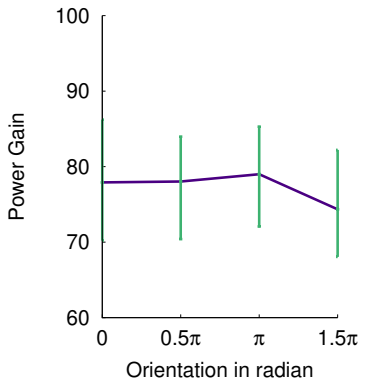

(b) Power Gain vs Orientation

Figure 10-Power gain vs receive antenna position in water. The figure shows the peak power gain for a 10-antenna CIB to a single antenna transmitter. Error bars represent $10^{t h}$ and $90^{t h}$ percentiles.

and (b) plot the median, $10^{t h}$ and $90^{t h}$ percentile of the power gain vs distance and orientation respectively. The figures show that the gain from IVN's beamformer remains stable across the different configurations and hence is independent of the distance and orientation of the receive antenna. This result is expected since CIB is inherently blind to channel conditions. It is important to note that while the gain is relatively uniform across different locations, the overall power received decreases with depth due to increased path loss.

(c) Gain vs Different Media: Next, we investigate whether IVN's power gains extend to different media. We repeat the same experiment as above, but this time we place the receive antenna in different media: air, water, $1 \mathrm{~L}$ of simulated gastric fluid [6], $1 \mathrm{~L}$ of simulated intestinal fluid [6], $20 \mathrm{~cm}$-thick steak, $20 \mathrm{~cm}$-thick bacon, and $20 \mathrm{~cm}$-thick chicken breast.

We compare IVN's gain to that of a baseline. The baseline also employs 10 antennas, similar to IVN's beamformer. However, since the transmitter does not know the channel, the baseline cannot focus its signal toward the receiver. ${ }^{5}$

We run 100 experiments in total. In each experiment, we change the location of the receive antenna. We plot the median, $10^{t h}$, and $90^{t h}$ percentile of the gain for both CIB and the baseline in Fig. 11. We make the following observations:

- IVN maintains its power gain across all the different media. Specifically, it achieves around $80 \times$ peak power boosting in comparison to a single-antenna transmitter.

- The baseline 10-antenna transmitter achieves a median gain of $10 \times$. This gain comes entirely from increasing the total amount of power transmitted. ${ }^{6}$

\footnotetext{
${ }^{5}$ We compared the performance of our baseline with coherent beamforming. Our results showed that while coherent beamforming outperforms our baseline transmitter in the air with line-of-sight testing, the performance difference is negligible across other media. This result is expected because coherent beamformers cannot capture the phase changes which RF signals experience as they travel across different media.

${ }^{6}$ Note that if we maintain the same power budget as a 1-antenna transmitter, it would only achieve $\sqrt{10}$ gain.
}

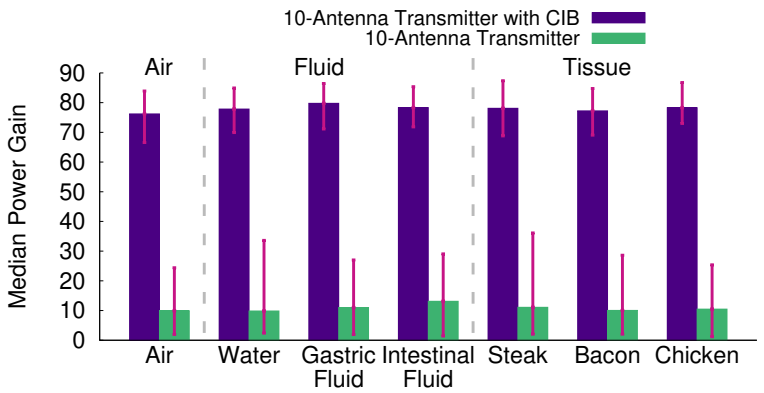

Figure 11-Gain across different media. The figure plots the gains of CIB (in purple) and a baseline 10-antenna beamformer (in green) over a singleantenna setup. Testing was performed in different types of simulated fluids and animal tissues. Error bars indicate $10^{t h}$ and $90^{t h}$ percentiles.

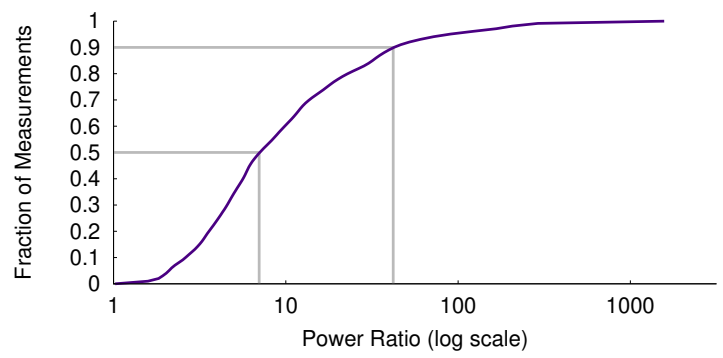

Figure 12-Gain of CIB over traditional coherent beamforming. The figure plots the CDF of the ratio of the received power using CIB to that using coherent beamforming, both with 10 -antenna transmitters.

- The gain is independent of the medium of operation. Recall from $\$ 3.7$ that this result is expected since CIB's gain is agnostic to the channel.

(d) Understanding the gain vs a 10-antenna baseline transmitter: Next, we ask whether IVN can outperform a 10 -antenna baseline at every single location. Hence, from every location measurement, we compute the ratio of the peak power of CIB to that of the baseline. Fig. 12 plots the CDF as a function of the power ratio in log scale. We make the following observations:

- CIB outperforms the baseline 10-antenna transmitter across over $99 \%$ of our experimental trials. This can be explained by multiple factors. First, the probability of the baseline having all its transmissions aligned at the receiver is exponentially low in the number of antennas. And second, CIB's frequencies are selected in a manner to maximize the expectation of the gain. Hence, the probability of constructive interference is extremely high by design.

- CIB achieves a median gain of around $8 \times$ over the baseline. This matches the results of Fig. 11.

- Finally, yet most importantly, CIB can achieve power gains more than $100 \times$ over the 10 -antenna transmitter in certain locations. The underlying reason is that a 10 -antenna transmitter may destructively interfere at various locations in 3D space. Unlike CIB, it has no mechanism of changing 


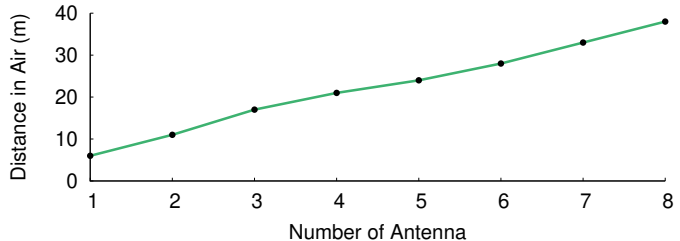

(a) Standard tag in air

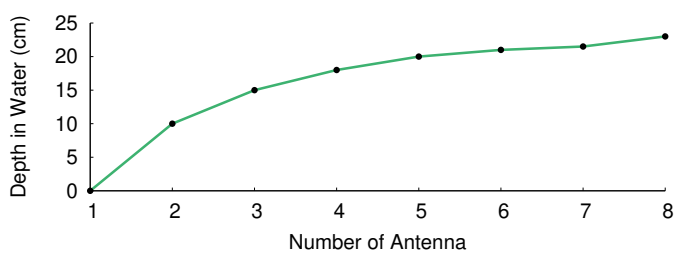

(a) Standard tag in Water

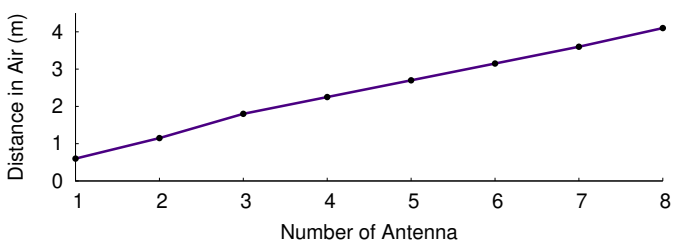

(b) Miniature tag in air

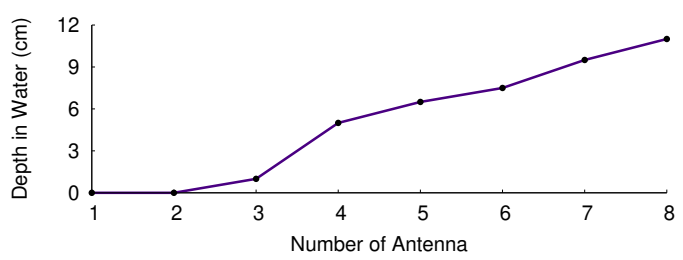

(b) Miniature tag in Water

Figure 13-Range vs number of antennas. We plot IVN's range as a function of antennas for standard and millimeter-sized tags both in air and in water.

its channel over time. Hence, in these scenarios, the 10antenna transmitter will have a power loss in comparison to a 1-antenna transmitter.

\subsubsection{IVN's Range Gain}

So far we have demonstrated that CIB delivers significant power gains even in blind channel conditions. Next, we evaluate the benefit of these gains in increasing the communication range (and depth) of battery-free RF sensors.

We consider two types of experimental environments, shown in Fig. 7 and Fig. 8. In the first setup, we are interested in evaluating the depth at which IVN allows us to power up and communicate with a sensor inside water. This evaluation is helpful for understanding in-vivo performance since biotissues have high water composition. Note that in this setup, the beamformer antennas are placed at a distance of $90 \mathrm{~cm}$ from the edge of the water tank. In the second setup, we are interested in evaluating the range at which IVN can power up and communicate with a sensor in air. We experiment with the two types of sensors described in $\S 5$ : a miniature sensor and a standard off-the-shelf RFID tag.

We perform 96 experimental trials in total. We vary the number of antennas used by IVN's beamformer and measure the maximum range of operation for both types of tags and in both evaluation setups. We determine the maximum range (depth) as the one where the reader can decode the tag's RN16. Once we have identified the maximum range, we repeat the experiment 3 times to ensure that the tag may still communicate.

Figs. 13(a)-(d) show our results. We plot the maximum range of the operation on the y-axis versus the number of antennas used by the beamformer on the X-axis. We make the following observations:

- The operation distance (range, depth) increases monotonically with the number of antennas. This increase is directly related to the peak power gain which enables the energy harvester to overcome its threshold voltage.

- For the in-air experiments, the maximum range for both types of tags increases by about $7.6 \times$ with 8 antennas. By comparison, the power gain from 8 antennas is around $55 \times$ as per Fig. 9. These two measurements are theoretically compatible because power decays quadratically with range in air; hence, the expected range gain is $\sqrt{55} \approx 7.4$.

- Considering the absolute distance numbers in line-of-sight also reveals an impressive performance. In particular, with 8 antennas, IVN can power up an RFID at a range $38 \mathrm{~m}$, while this range is only $5.2 \mathrm{~m}$ with a single antenna. This result has implications beyond in-vivo communication and holds the potential to advance RFID-based services like localization and inventory control.

- Most importantly, the in-water experiments demonstrate that IVN can provide substantial benefits. In particular, without CIB beamforming, neither the small nor the standard tag can be powered up. However, with an 8-antenna CIB prototype, we can communicate with a standard tag at $23 \mathrm{~cm}$ depth and with a miniature tag at $11 \mathrm{~cm}$ depth.

- Finally, note that the relationship between the depth and antenna number is logarithmic, which is expected since RF signals experience exponential loss vs depth in water.

\subsection{In-Vivo Evaluation}

Finally, we conduct in-vivo experiments to evaluate IVN's ability to power up and communicate with implantable battery-free sensors.

Animal Preparation: All procedures were conducted in accordance with protocols approved by our institution's Committee on Animal Care. In vivo porcine studies were performed in a female Yorkshire pig weighing $85 \mathrm{Kg}$. The pig was fasted overnight prior to the procedure. Sedation 


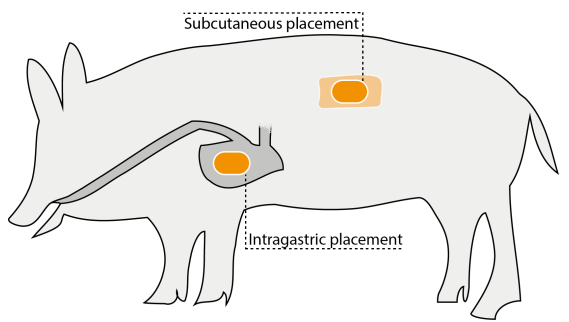

Figure 14 - Swine Experiment. Battery-free tags were implanted inside a living swine to test IVN's ability to deliver power and communicate. We evaluated subcutaneous (under the skin) and intragastric (inside the stomach) placements.

was performed by intramuscular injection of Telazol (tiletamine/zolazepam) $5 \mathrm{mg} / \mathrm{kg}$, xylazine $2 \mathrm{mg} / \mathrm{kg}$, and atropine $0.05 \mathrm{mg} / \mathrm{kg}$, prior to intubation. Anesthesia was maintained with isoflurane at $1-3 \%$. A laparotomy was performed using a ventral middle line incision from the xiphoid to the pubis.

Methods: We evaluate IVN with sensors implanted in the two locations shown in Fig. 14:

- In the first set of experiments the standard tag (placed in $15 \mathrm{~mL}$ falcon tube) as well as the miniature tag (placed in a plastic tube, $\mathrm{dm}: 1 \mathrm{~cm}$ ) were placed in the stomach through a $3 \mathrm{~cm}$ incision. Readings were taken with all antennas being positioned $30-80 \mathrm{~cm}$ lateral (left side) to the animal such that they were in line with the coronal plane.

- Following euthanization, subcutaneous performance of both devices was evaluated by subcutaneous placement (left side, placement and reading as described above).

To evaluate reproducibility, all placements were performed at least three times. In each experiment, we remove the RFID and place it back, changing its location and orientation inside the swine's body.

To evaluate whether IVN is able to power up the RFID, we correlate the responses with the RFID's known 12-bit preamble "110100100011" (FM0 encoding). Communication is successful if the highest correlation is larger than 0.8.

Results: We summarize our results below:

- In the gastric placement, IVN could establish communication with the standard tag in half of the six experimental trials. A sample output from these experiments is shown in Fig. 15(a), where it shows the captured waveform and the decoded bits. This result demonstrates that IVN is indeed capable of powering up and communicating through deeptissues. We suspect that in the experiments where we could not establish a communication session, the tag may have moved due to the swine's breathing motion or it may have been misoriented with respect to CIB's antennas.

- IVN was unable to establish communication with the miniature tag when placed inside the stomach. This can be explained by the fact that the tag's miniature antenna precluded harvesting sufficient energy for powering up.

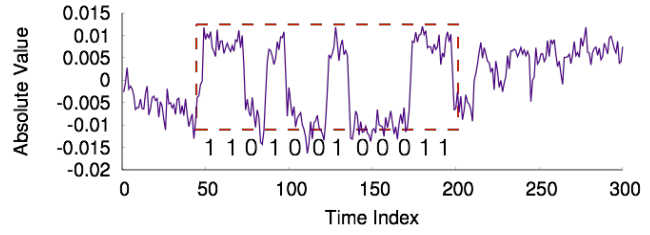

(a) Standard tag placed in stomach

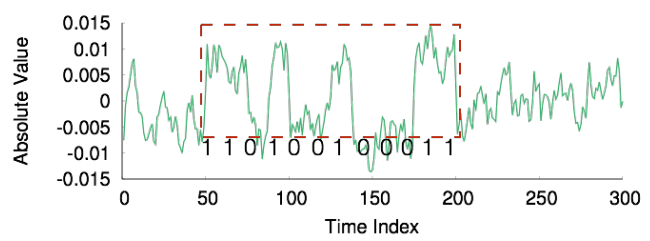

(b) Miniature tag placed subcutaneously

Figure 15-Measured response from implantable sensors. We plot the time-domain signals received from the sensors and the decoded bits.

- In the subcutaneous evaluation, IVN was successful in powering up the standard and miniature tag across all the experimental trials. A sample output is shown in Fig. 15(b) demonstrating the tag's response.

These results demonstrate the potential of IVN for enabling in-vivo networking.

\section{RELATED WORK}

IVN is related to prior work in three areas: energy harvesting bioelectronics, beamforming algorithms, and backscatter networking. In contrast to past work in these areas, IVN is the first to bring the benefits of MIMO beamforming to in-vivo battery-free sensors. This enables powering up and communicating with deep-tissue miniature implantable devices, without requiring the transmitter to be in direct contact or close proximity to the body.

Energy-harvesting bioelectronics. Past work in this area broadly falls in two main categories: self-powered and wirelessly-powered implants. Self-powered devices harvest energy from the body using thermoelectric [29], piezoelectric [20,65, 68], biopotential [47], or glucose [15, 53] power extraction techniques. While attractive, these techniques suffer from being anatomically specific. For example, piezoelectric harvesters are most efficient near joints where there is more motion but they are less effective in internal organs where their ability to harness power is limited to sub$\mu \mathrm{W} / \mathrm{mm}^{2}$ [27].

The desire to create battery-free devices that are not limited to specific regions of the body has led researchers to explore wireless power transfer $[12,38]$. The majority of wirelessly powered implantables rely on near-field coupling [55], where a coil inserted inside a mammal can be wirelessly powered from a transmit coil right outside the mammal's body. Such power delivery has been demonstrated for cochlear implants [48] and RFIDs implanted under the skin [17]. The 
main challenge with this approach, however, is that it suffers from a large coil-to-distance ratio [64]. Said differently, it either requires implanting large coils with a diameter of few centimeters [13] or it can only operate at very superficial depths $[50,64]$. This is why practical implementations that leverage this technology for millimeter-size devices are limited to depths smaller than $1 \mathrm{~cm}[11,27]$.

To enable deep-tissue power delivery, researchers have recently proposed new approaches like midfield $[9,50]$ and ultrasonic [14] power transfer. While these systems can achieve greater depths, they still constrain the distance between the transmitting coil/radiator and the body instrumented with the implantable. A state-of-the-art midfield device, for example, can only operate if a mouse is placed within a RF resonant cavity of $10 \mathrm{~cm}$ diameter [50]. Other proposals using midfield [9] and ultrasonic powering [14] require direct contact between the transmitter and the human body.

IVN's fundamental contribution, CIB, is orthogonal to these recent advances in wireless power delivery. In particular, by demonstrating how one could beamform transmissions from multiple antennas to a battery-free device without channel knowledge, IVN can deliver power to deep tissues $(>10 \mathrm{~cm})$, even if the transmitter is placed more than a meter away from the body. While our particular implementation has been demonstrated in the far field, the algorithmic framework may be extended to other domains like the midfield by incorporating their respective channel models and devices.

Beamforming algorithms. Beamforming algorithms leverage multiple transmit antennas (or coils) to focus their energy toward a receiver of interest. Existing beamforming algorithms fall into three main categories: MIMO, antenna array, and magnetic. MIMO beamforming focuses energy by inverting the estimated channel between the transmitter and the receiver [24]; however, channel estimates can only be obtained if the receiver is powered up in the first place. Hence, while it has been used extensively in wireless communications, it is not applicable for battery-free devices. Antenna-array beamforming operates by precoding its transmissions to focus energy in a specific spatial direction or angle [18, 33, 62, 67]. While such precoding can be easily derived in homogeneous media, it becomes intractable with multi-layer tissues, making this technique impractical for in-vivo devices. Finally, MagMIMO is a recently proposed technology that can beamform in the near field [25, 32]. Near-field powering, however, suffers from a large coil size-to-distance ratio as mentioned above. Hence, the technology is more applicable to cellphones which can incorporate larger coils than to deep-tissue power delivery to miniature implantable devices.

IVN's beamformer is inspired by seminal work in the areas of blind source separation (often called algebraic blind beamforming) $[18,56,62]$ and opportunistic beamforming [58, 63], which aim to operate under blind or partially-blind channel conditions. In contrast to IVN, however, prior techniques require cooperation from a receiver - which needs to be powered up in the first place - in order to realize their beamforming gains. In particular, they either require channel feedback from the receiver (for opportunistic beamforming) or require the receiver to manipulate the received signal (for blind source separation). While IVN is motivated by these techniques, it can realize beamforming gains without any receiver cooperation, enabling it to operate with battery-free sensors. To do so, it formulates a new optimization problem that maximizes peak power across space, while accounting for communication constraints of backscatter designs. As a result, it demonstrates the first blind beamformer that can power up and communicate with miniature battery-free devices inside living mammals.

Backscatter Networking. Recently, the topic of backscatter networking has garnered significant attention from the networking community [30, 43-45, 69, 72]. Much research has been done to increase the throughput $[70,71]$ and coverage [60] of these systems and enable them to backscatter different types of signals like WiFi [35], Bluetooth [31], and TV signals [43]. These systems rely on RF power harvesting in the far-field, and hence still need to receive sufficient power from a transmitter in order to power up. State-of-the-art systems like HitchHike [30, 43, 69] all require the batteryfree node to be placed within 3-8 $\mathrm{m}$ from the transmitter to harness enough power. This range limitation is similar to RFIDs. LoRa backscatter can achieve larger range but it requires a $10 \mathrm{~cm}^{2}$ battery [59]. Note that some backscatter systems can operate at a larger distance by harnessing power from TV stations that broadcast MegaWatts of power [43] and by leveraging large $6 \mathrm{dBi}$ antennas [52]. Naturally, however, bringing megaWatt transmitters indoors to power up in deep tissue is neither FCC compliant nor safe for human exposure [50]. IVN is motivated by these recent advances but is fundamentally orthogonal to them. In particular, by enabling blind-channel beamforming, it brings the benefits of MIMO beamforming for battery-free backscatter nodes. Moreover, its intrinsic duty-cycled operation makes it FCC compliant and safe for human exposure. Hence, it presents the first system that can enable communication with backscatter nodes in deep tissues.

Acknowledgments. We thank Siddartha Tamang, Joy Collins, and Alison Hayward from the MIT animal facility and the MIT Koch Institute for their support with the animal trials. We also thank our shepherd, Haitham Hassanieh, and the anonymous SIGCOMM reviewers for their feedback and insights. This research is partially supported by the MIT Media Lab, the NSF, and the NIH. Christoph Steiger is supported in part by Feodor Lynen Fellowship from the Alexander von Humboldt Foundation. 


\section{REFERENCES}

[1] Avery denison. http://rfid.averydennison.com. Avery Denison.

[2] CDA2990. http://www.ettus.com. Ettus Inc.

[3] EPC UHF Gen2 Air Interface Protocol. http://www.gs1.org/epcrfid/ epc-rfid-uhf-air-interface-protocol/2-0-1.

[4] MTI RFID antenna. http://www.mtiwe.com. MTI Wireless Edge.

[5] SBX daughterboard. http://www.ettus.com. ettus inc.

[6] United states pharmacopeia (usp) 37. USP.

[7] usrp n210. http://www.ettus.com. ettus inc.

[8] Xerafy. http://www.xerafy.com. Xerafy.

[9] Abid, A., O’Brien, J. M., Bensel, T., Cleveland, C., Booth, L., Smith, B. R., LANGer, R., AND Traverso, G. Wireless power transfer to millimeter-sized gastrointestinal electronics validated in a swine model. Scientific reports 7 (2017), 46745.

[10] Anikeeva, P., Andalman, A. S., Witten, I., Warden, M., Goshen, I., Grosenick, L., Gunaydin, L. A., Frank, L. M., AND DEISSEROTH, K. Optetrode: a multichannel readout for optogenetic control in freely moving mice. Nature neuroscience 15, 1 (2012), 163-170.

[11] Arbabian, A., Chang, T. C., Wang, M. L., Charthad, J., BaltSavias, S., Fallahpour, M., AND Weber, M. J. Sound technologies, sound bodies: Medical implants with ultrasonic links. IEEE Microwave Magazine 17, 12 (2016), 39-54.

[12] Assawaworrarit, S., YU, X., AND Fan, S. Robust wireless power transfer using a nonlinear parity-time-symmetric circuit. Nature 546, 7658 (2017), 387.

[13] Carta, R., Tortora, G., Thoné, J., Lenaerts, B., Valdastri, P., Menciassi, A., Dario, P., AND Puers, R. Wireless powering for a self-propelled and steerable endoscopic capsule for stomach inspection. Biosensors and Bioelectronics 25, 4 (2009), 845-851.

[14] Charthad, J., Weber, M. J., Chang, T. C., And Arbabian, A. A mm-sized implantable medical device (imd) with ultrasonic power transfer and a hybrid bi-directional data link. IEEE Journal of solid-state circuits 50, 8 (2015), 1741-1753.

[15] Chaudhuri, S. K., And Lovley, D. R. Electricity generation by direct oxidation of glucose in mediatorless microbial fuel cells. Nature biotechnology 21, 10 (2003), 1229.

[16] Chen, Y., Chiotellis, N., Chuo, L.-X., Pfeiffer, C., Shi, Y., Dreslinski, R. G., Grbic, A., Mudge, T., Wentzloff, D. D., BlAAUW, D., ET AL. Energy-autonomous wireless communication for millimeter-scale internet-of-things sensor nodes. IEEE Journal on Selected Areas in Communications 34, 12 (2016), 3962-3977.

[17] Chrysler, A., Furse, C., And Chung, Y. Biocompatible, implantable uhf rfid antenna made from conductive ink. In Antennas and Propagation (APSURSI), 2016 IEEE International Symposium on (2016), IEEE, pp. 467-468.

[18] Coviello, C., AND Sibul, L. Blind source separation and beamforming: algebraic technique analysis. IEEE Transactions on Aerospace and Electronic Systems 40, 1 (2004), 221-235.

[19] Dagdeviren, C. The future of bionic dynamos. Science 354, 6316 (2016), 1109-1109.

[20] Dagdeviren, C., Yang, B. D., Su, Y., Tran, P. L., Joe, P., Anderson, E., Xia, J., Doraiswamy, V., Dehdashti, B., Feng, X., ET AL. Conformal piezoelectric energy harvesting and storage from motions of the heart, lung, and diaphragm. Proceedings of the National Academy of Sciences 111, 5 (2014), 1927-1932.

[21] Deisseroth, K. Optogenetics. Nature methods 8, 1 (2011), 26-29.

[22] DiCKSON, J. F. On-chip high-voltage generation in mnos integrated circuits using an improved voltage multiplier technique. IEEE Journal of solid-state circuits 11, 3 (1976), 374-378.
[23] Dove, I. Analysis of radio propagation inside the human body for in-body localization purposes. Master's thesis, University of Twente, 2014.

[24] Halperin, D., Hu, W., Sheth, A., AND Wetherall, D. 802.11 with multiple antennas for dummies. ACM SIGCOMM Computer Communication Review 40, 1 (2010), 19-25.

[25] Hamed, E., Rahul, H., Abdelghany, M. A., and Katabi, D. Real-time distributed mimo systems. In Proceedings of the 2016 ACM SIGCOMM Conference (2016), ACM, pp. 412-425.

[26] Hameed, Z., AND MoEZ, K. Hybrid forward and backward thresholdcompensated rf-dc power converter for rf energy harvesting. IEEE Journal on Emerging and Selected Topics in Circuits and Systems 4, 3 (2014), 335-343.

[27] Hannan, M. A., Mutashar, S., Samad, S. A., and Hussain, A. Energy harvesting for the implantable biomedical devices: issues and challenges. Biomedical engineering online 13, 1 (2014), 79.

[28] Ho, J. S., Yeh, A. J., Neofytou, E., Kim, S., Tanabe, Y., PatLOLLA, B., BEYGUI, R. E., AND PoOn, A. S. Wireless power transfer to deep-tissue microimplants. Proceedings of the National Academy of Sciences 111, 22 (2014), 7974-7979.

[29] Hochbaum, A. I., Chen, R., Delgado, R. D., Liang, W., GarNETT, E. C., NAJARIAN, M., MAJUMdar, A., AND YANG, P. Enhanced thermoelectric performance of rough silicon nanowires. Nature 451,7175 (2008), 163.

[30] Iyer, V., Talla, V., Kellogg, B., Gollakota, S., and Smith, J. Inter-technology backscatter: Towards internet connectivity for implanted devices. In Proceedings of the 2016 ACM SIGCOMM Conference (2016), ACM, pp. 356-369.

[31] Iyer, V., Talla, V., Kellogg, B., Gollakota, S., and Smith, J. Inter-technology backscatter: Towards internet connectivity for implanted devices. In Proceedings of the 2016 ACM SIGCOMM Conference (2016), ACM, pp. 356-369.

[32] Jadidian, J., AND KatABI, D. Magnetic mimo: How to charge your phone in your pocket. In Proceedings of the 20th annual international conference on Mobile computing and networking (2014), ACM, pp. 495506.

[33] Joshi, K. R., Hong, S. S., And Katti, S. Pinpoint: Localizing interfering radios. In NSDI (2013), pp. 241-253.

[34] Kargas, N., Mavromatis, F., AND Bletsas, A. Fully-coherent reader with commodity sdr for gen $2 \mathrm{fm} 0$ and computational rfid. IEEE Wireless Communications Letters 4, 6 (2015), 617-620.

[35] Kellogg, B., Parks, A., Gollakota, S., Smith, J. R., and WETHERALL, D. Wi-fi backscatter: Internet connectivity for rfpowered devices. In ACM SIGCOMM Computer Communication Review (2014), vol. 44, ACM, pp. 607-618.

[36] KIM, C. W., AND SEE, T. S. P. Rf transmission power loss variation with abdominal tissues thicknesses for ingestible source. In E-Health Networking Applications and Services (Healthcom), 2011 13th IEEE International Conference on (2011), IEEE, pp. 282-287.

[37] Kim, J., Banks, A., Xie, Z., Heo, S. Y., Gutruf, P., LeE, J. W., XU, S., JANG, K.-I., LiU, F., Brown, G., ET AL. Miniaturized flexible electronic systems with wireless power and near-field communication capabilities. Advanced Functional Materials 25, 30 (2015), 4761-4767.

[38] Kurs, A., Karalis, A., Moffatt, R., Joannopoulos, J. D., FISHER, P., AND SOLJACIĆ, M. Wireless power transfer via strongly coupled magnetic resonances. science 317, 5834 (2007), 83-86.

[39] Kurup, D., Joseph, W., Vermeeren, G., and Martens, L. Inbody path loss model for homogeneous human tissues. IEEE Transactions on Electromagnetic Compatibility 54, 3 (2012), 556-564.

[40] LAZZI, G. Thermal effects of bioimplants. IEEE Engineering in Medicine and Biology Magazine 24, 5 (2005), 75-81. 
[41] Le, T., Mayaram, K., And Fiez, T. Efficient far-field radio frequency energy harvesting for passively powered sensor networks. IEEE Journal of solid-state circuits 43, 5 (2008), 1287-1302.

[42] Lee, C. H., Kim, H., Harburg, D. V., Park, G., Ma, Y., Pan, T., KIM, J. S., LEE, N. Y., KIM, B. H., JANG, K.-I., ET AL. Biological lipid membranes for on-demand, wireless drug delivery from thin, bioresorbable electronic implants. NPG Asia materials 7 (2015).

[43] LiU, V., Parks, A., Talla, V., Gollakota, S., Wetherall, D., AND SMITH, J. R. Ambient backscatter: wireless communication out of thin air. ACM SIGCOMM Computer Communication Review 43, 4 (2013), 39-50.

[44] MA, Y., HUI, X., AND KAN, E. C. 3d real-time indoor localization via broadband nonlinear backscatter in passive devices with centimeter precision. In Proceedings of the 22nd Annual International Conference on Mobile Computing and Networking (2016), ACM, pp. 216-229.

[45] MA, Y., Selby, N., AND AdiB, F. Drone relays for battery-free networks. In Proceedings of the Conference of the ACM Special Interest Group on Data Communication (2017), ACM, pp. 335-347.

[46] Ma, Y., Selby, N., AND AdIB, F. Minding the billions: Ultrawideband localization for deployed rfid tags. In Proceedings of the $23 \mathrm{rd}$ Annual International Conference on Mobile Computing and Networking (2017), ACM, pp. 248-260.

[47] Mercier, P. P., Lysaght, A. C., Bandyopadhyay, S., ChanDRAKASAN, A. P., AND STANKOVIC, K. M. Energy extraction from the biologic battery in the inner ear. Nature biotechnology 30, 12 (2012), 1240 .

[48] Mercier, P. P., Lysaght, A. C., Bandyopadhyay, S., ChanDRAKASAN, A. P., AND STANKOVIC, K. M. Energy extraction from the biologic battery in the inner ear. Nature biotechnology 30, 12 (2012), 1240.

[49] MerLI, F. Implantable antennas for biomedical applications.

[50] Montgomery, K. L., YeH, A. J., Ho, J. S., TsaO, V., IYer, S. M., Grosenick, L., Ferenczi, E. A., Tanabe, Y., Deisseroth, K., DELP, S. L., ET AL. Wirelessly powered, fully internal optogenetics for brain, spinal and peripheral circuits in mice. nAture methods (2015).

[51] NiKitin, P. V., AND RAO, K. S. Antennas and propagation in uhf rfid systems. In RFID, 2008 IEEE International Conference On (2008), IEEE, pp. 277-288.

[52] Parks, A. N., Sample, A. P., Zhao, Y., And Smith, J. R. A wireless sensing platform utilizing ambient rf energy. In Biomedical Wireless Technologies, Networks, and Sensing Systems (BioWireleSS), 2013 IEEE Topical Conference on (2013), IEEE, pp. 154-156.

[53] Rapoport, B. I., KedZierski, J. T., And SarpeshKar, R. A glucose fuel cell for implantable brain-machine interfaces. PloS one 7 , 6 (2012), e38436

[54] Sauer, C., Stanacevic, M., Cauwenberghs, G., and Thakor, N. Power harvesting and telemetry in cmos for implanted devices. IEEE Transactions on Circuits and Systems I: Regular Papers 52, 12 (2005), 2605-2613

[55] Seo, Y.-S., Hughes, Z., Isom, D., Nguyen, M. Q., Deb, S., Rao, S., AND CHIAO, J.-C. Wireless power transfer for a miniature gastrostimulator. In Microwave Conference (EuMC), 2012 42nd European (2012), IEEE, pp. 229-232.

[56] Sidiropoulos, N. D., AND LiU, X. Identifiability results for blind beamforming in incoherent multipath with small delay spread. IEEE
Transactions on Signal Processing 49, 1 (2001), 228-236.

[57] Sumansin, W., Phasukkit, P., Pintavirooj, C., and SanPANICH, A. Analysis of heat transfer and specific absorption rate of electromagnetic field in human body at $915 \mathrm{mhz}$ and $2.45 \mathrm{ghz}$ with 3d finite element method. In Biomedical Engineering International Conference (BMEiCON), 2012 (2012), IEEE, pp. 1-4.

[58] Svedman, P., Wilson, S. K., Cimini, L. J., and Ottersten, B. Opportunistic beamforming and scheduling for ofdma systems. IEEE Transactions on Communications 55, 5 (2007), 941-952.

[59] Talla, V., Hessar, M., Kellogg, B., Najafi, A., Smith, J. R., AND GOLLAKOTA, S. Lora backscatter: Enabling the vision of ubiquitous connectivity. Proceedings of the ACM on Interactive, Mobile, Wearable and Ubiquitous Technologies 1, 3 (2017), 105.

[60] Talla, V., KellogG, B., Ransford, B., NAderiparizi, S., GolLAKOTA, S., AND SMITH, J. R. Powering the next billion devices with wi-fi. In Proceedings of the 11th ACM Conference on Emerging Networking Experiments and Technologies (2015), ACM, p. 4.

[61] Traverso, G., Ciccarelli, G., Schwartz, S., Hughes, T., Boettcher, T., Barman, R., Langer, R., and Swiston, A. Physiologic status monitoring via the gastrointestinal tract. PloS one 10, 11 (2015), e0141666.

[62] Van Der Veen, A.-J. Algebraic methods for deterministic blind beamforming. Proceedings of the IEEE 86, 10 (1998), 1987-2008.

[63] Viswanath, P., Tse, D. N. C., and Laroia, R. Opportunistic beamforming using dumb antennas. IEEE transactions on information theory 48, 6 (2002), 1277-1294.

[64] WAFFEnschmidT, E., AND STARING, T. Limitation of inductive power transfer for consumer applications. In Power Electronics and Applications, 2009. EPE'09. 13th European Conference on (2009), IEEE, pp. 1-10.

[65] WANG, Z. L., AND Wu, W. Nanotechnology-enabled energy harvesting for self-powered micro-/nanosystems. Angewandte Chemie International Edition 51, 47 (2012), 11700-11721.

[66] Warden, M. R., Cardin, J. A., And Deisseroth, K. Optical neural interfaces. Annual review of biomedical engineering 16 (2014), 103-129.

[67] Xiong, J., And Jamieson, K. ArrayTrack: a fine-grained indoor location system. In Usenix NSDI (2013).

[68] Xu, S., Hansen, B. J., AND Wang, Z. L. Piezoelectric-nanowireenabled power source for driving wireless microelectronics. Nature communications 1 (2010), 93.

[69] Zhang, P., Bharadia, D., Joshi, K., And Katti, S. Hitchhike: Practical backscatter using commodity wifi. In Proceedings of the 14th ACM Conference on Embedded Network Sensor Systems CD-ROM (2016), ACM, pp. 259-271.

[70] Zhang, P., AND GANESAN, D. Enabling bit-by-bit backscatter communication in severe energy harvesting environments. In NSDI (2014), pp. 345-357.

[71] Zhang, P., Hu, P., Pasikanti, V., and Ganesan, D. Ekhonet: High speed ultra low-power backscatter for next generation sensors. In Proceedings of the 20th annual international conference on Mobile computing and networking (2014), ACM, pp. 557-568.

[72] Zhang, P., Rostami, M., Hu, P., AND GANesan, D. Enabling practical backscatter communication for on-body sensors. In Proceedings of the 2016 ACM SIGCOMM Conference (2016), ACM, pp. 370-383. 